\title{
Inverted Variant of Takotsubo Syndrome Caused by Inhaled Adrenergic Beta-2 agonists
}

\author{
Marta de Sousa ${ }^{1}$, André Casado ${ }^{2}$, Alexandre Buinhas Marques², Francisco Pereira Machado ${ }^{3}$, Isabel Esperança² \\ ${ }^{1}$ Internal Medicine Department, Centro Hospitalar do Médio Tejo, E.P.E., Torres Novas, Portugal \\ ${ }^{2}$ Intensive Care Unit, Hospital da Luz, Lisbon, Portugal \\ ${ }^{3}$ Cardiology Department, Hospital da Luz, Lisbon, Portugal
}

Received: 22/12/2017

Accepted: $22 / 01 / 2018$

Published: $20 / 02 / 2018$

How to cite this article: de Sousa M, Casado A, Marques A, Machado F, Esperança I. Inverted variant of Takostubo syndrome caused by inhaled adrenergic beta-2 agonists. EJCRIM 2018;5: doi:10.12890/2018_000831.

Conflicts of Interests: The Authors declare that there are no competing interests.

This article is licensed under a Commons Attribution Non-Commercial 4.0 License

\section{ABSTRACT}

Takotsubo syndrome (TS) is an acute and reversible clinical syndrome characterized by transient hypokinesis of the left ventricular (LV) apex. Variant forms of LV dysfunction have been reported, including inverted Takotsubo syndrome (ITS), which represents only $5 \%$ of cases and has previously been linked to excessive use of inhaled adrenergic beta-2 agonists. The authors describe the case of a 60 -year-old female patient who was diagnosed with ITS after the excessive use of inhaled adrenergic beta- 2 agonists. This case highlights an uncommon variant of this syndrome that may not be obvious and must be suspected in this particular context.

\section{LEARNING POINTS}

- Takotsubo syndrome (TS) was initially described with a classic pattern of LV apical akinesis and accounts for around 75-80\% of cases. Variants including inverted Takotsubo (also known as basal variant) can affect other areas of the myocardium.

- Several physiopathological mechanisms have been implicated. Catecholamine-induced cardiotoxicity is one of the most supported theories, while other triggers, including excessive use of inhaled beta- 2 agonists, have also been described.

- Treatment of TS is mainly symptomatic and conservative and frequently leads to rapid resolution and LV function recovery.

\section{KEYWORDS}

Takotsubo syndrome, catecholamines, adrenergic beta- 2 agonists

\section{CASE REPORT}

We report the case of a 60-year-old woman with a history of chronic obstructive pulmonary disease (COPD), hypereosinophilic syndrome, hypothyroidism, rheumatoid arthritis, depression and arterial hypertension, whose treatment included fluticasone/salmeterol $50 \mu \mathrm{g} / 250$ $\mu g$ two puffs twice a day. The patient presented to the emergency room (ER) with a depressed level of consciousness after experiencing dyspnoea, cough and fever for 2 days at home. At home the patient had already overused her bronchodilator, but at the ER she received continuous nebulized salbutamol, furosemide and intravenous hydrocortisone, with no clinical improvement and a worsening of respiratory acidosis. The patient was promptly transferred to the ICU for intubation and mechanical ventilation under ketamine sedation, due to severe bronchospasm. Blood tests revealed an elevated CRP (13.17 mg/dl) and elevated NT-proBNP (11695 pg/ml), while the initial set of cardiac 
markers was normal but rose subsequently, with a troponin I peak of $9.18 \mathrm{ng} / \mathrm{ml}$. The electrocardiogram showed ST depression in leads V2 and V3, while the echocardiogram showed depressed systolic function and akinesis in the mid and basal segments. A coronary angiogram demonstrated normal epicardial coronary arteries with normal TIMI 3 flow. Ventriculography revealed a mid-wall and basal ballooning pattern, suggesting the diagnosis of inverted Takotsubo syndrome (ITS) (Fig. 1).

A thorax CT scan showed diffuse ground glass opacification and so antibiotherapy with piperacilin-tazobactam and azithromycin was started. Evolution was favourable, leading to extubation within 3 days. A subsequent echocardiogram, performed 3 days after the angiogram, showed complete recovery of left ventricular function with preserved ejection fraction and normal wall motion. The patient was discharged from the ICU at that point. The echocardiogram remained normal 5 months later.

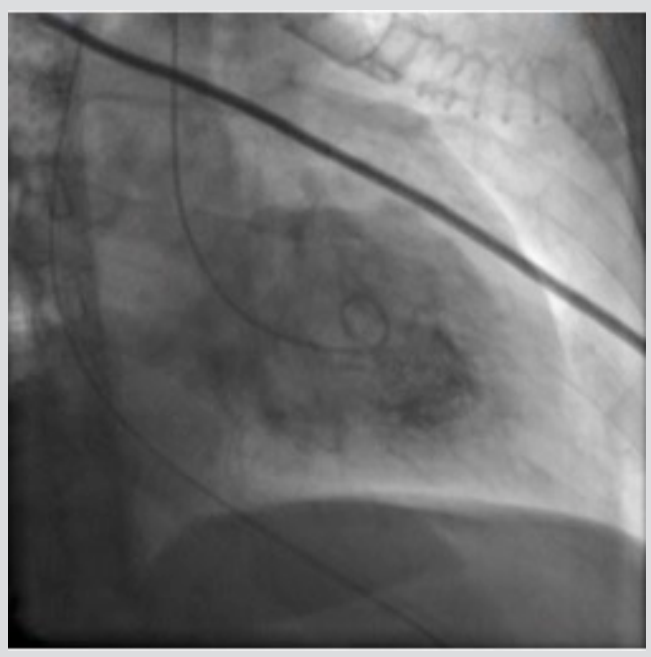

Figure 1.Ventriculographic appearance showing a mid-wall and basal ballooning pattern, suggesting the diagnosis of inverted Takotsubo syndrome

\section{DISCUSSION}

We report this clinical case because Takotsubo syndrome (TS) is a relatively recent cardiac diagnosis and remains a clinical challenge, especially in the uncommon variants like ITS. Its true prevalence remains unknown ${ }^{[1]}$. TS represents $1-2 \%$ of suspected cases of acute coronary syndrome and is a very important differential diagnosis of acute myocardial infarction ${ }^{[1,2]}$. Prompt diagnosis is extremely important as both the treatment and prognosis of these clinical entities differ significantly. The condition predominantly affects postmenopausal women $^{[2]}$ (more than $90 \%$ of cases) and the major symptoms may include chest pain and dyspnoea, with electrocardiographic changes, such as ST-segment depression, as in the presented case ${ }^{[3,4]}$, but which may be indistinguishable from typical ST-elevation myocardial infarction. The diagnosis of TS requires the exclusion of obstructive coronary artery disease, and thus an emergent angiogram is crucial in order, for instance, to avoid unnecessary thrombolysis ${ }^{[1]}$, as well as other unwarranted treatments. All these features are part of the latest Takotsubo Syndrome Diagnostic Criteria adopted in 2015 by the Heart Failure Association of the European Society of Cardiology ${ }^{[3]}$.

The name 'Takotsubo' refers to the originally described, and hence classic, form of extensive apical and/or mid-venticular akinesia or hypokinesia and basal hypercontractility (narrow base with apical ballooning), resembling the octopus-catching vases of Japanese fisherman ${ }^{[1,3]}$. However, there are anatomical variants, including ITS which is also known as the basal variant and which represents $5 \%$ of all TS, with circumferential basal hypokinesia and apical hypercontractility ${ }^{[1,3]}$. There are other anatomical variants, such as the mid left ventricular variant (the most frequent variant), biventricular apical dysfunction, isolated right ventricular or dysfunction sparing apical tip, with uncertain prevalence. A likely explanation for the variants is the different rates of LV segment recovery ${ }^{[3]}$.

Possible pathophysiological mechanisms have been discussed including coronary microvascular dysfunction, coronary artery spasm ${ }^{[5]}$ and abnormalities in cardiac fatty acid metabolism, but catecholamine-induced cardiotoxicity is currently the most supported explanation ${ }^{[2]}$. Increased beta-2-adrenoreceptor stimulation has been proposed as a possible cause of this entity ${ }^{[6]}$ and the task force on TS of the Heart Failure Association of the European Society of Cardiology proposed a classification as secondary TS ${ }^{[3]}$ as a substantial proportion of cases occur in patients already hospitalized for another medical condition, and are a complication of the primary condition or its treatment ${ }^{[3]}$. Our patient can be diagnosed as having secondary TS since she was hospitalized due to acute exacerbation of COPD. It is probable that treatment with large doses of inhaled adrenergic beta- 2 agonists, associated with activation of the sympathetic nervous system and a rise 
in catecholaminergic tone, precipitated acute TS. Emotional stress and illicit drug abuse were ruled out. The latest recommendations also suggest that after diagnosis, patients should be triaged according to a risk stratification algorithm; according to current recommendations, this patient would have been included in the high-risk category. All patients with TS should be monitored in a coronary care/high-dependency unit for 24 hours. After that, according to risk stratification, high-risk patients should stay in these units, while low-risk patients may be transferred to lower level monitoring units. However, this system is currently based on expert opinion (with a level of evidence of C), due to the lack of prospective studies ${ }^{[3]}$.

Treatment of this condition during the acute phase is mainly symptomatic and - as in the case of our patient - management should focus on the triggering underlying condition ${ }^{[2]}$. The role of beta-blockers is still controversial but may provide some protection in selected patients, such as those with recurrent $\mathrm{TS}^{[3]}$. In the ER, beta- 2 agonists should be used with caution and the recommended dose should not be exceeded. Treatment of bronchospasm with beta- 2 agonists can be avoided as anticholinergic agents, steroids and mast cell stabilizers can be used instead.

As in our patient, prognosis is generally good; left ventricular function starts to recover within a few days and is usually complete within 3-4 weeks ${ }^{[4]}$. The authors believe that this clinical case report contributes further to the knowledge of this entity and highlights a rare form of this syndrome.

\section{REFERENCES}

1. Nóbrega S, Brito D. The "broken heart syndrome": state of the art. Rev Port Cardiol 2012;31:589-596.

2. Komamura K, Fukui M, Iwasaku T, Hirotani S, Masuyama T. Takotsubo cardiomyopathy: pathophysiology, diagnosis and treatment. World J Cardiol 2014;6:602-609.

3. Lyon A, Bossone E, Schneider B, Sechtem U, Citro R, Underwood S, et al. Current state of knowledge on Takotsubo syndrome: a position statement from the task force on Takotsubo syndrome of the Heart Failure Association of the European Society of Cardiology. Eur J Heart Fail 2016;18:8-27.

4. Roshanzamir S, Showkathali R. Takotsubo cardiomyopathy a short review. Curr Cardiol Rev 2013;9:191-196.

5. Dores H, Raposo L, Ferreira J, Andrade MJ, Almeida M, Mendes M. Apical ballooning syndrome during diagnostic coronary angiography. Arq Bras Cardiol 2013;100:e47-50.

6. Mendonza I, Novaro G. Repeat recurrence of takotsubo cardiomyopathy related to inhaled beta-2-adrenoceptor agonists. World J Cardiol 2012;4:211-213. 